\title{
Interacción social entre los niños y niñas con necesidades educativas especiales y sus pares: una revisión narrativa
}

\author{
Angelita Gabriela Arroyo Limaico \\ Lorena Toro-Mayorga \\ Universidad Técnica del Norte \\ agarroyol@utn.edu.ec
}

\section{RESUMEN}

Este estudio analiza las relaciones sociales entre los niños y niñas con Necesidades Educativas Especiales (NEE) y sus pares desde una revisión narrativa de artículos vinculados al tema. La estrategia de búsqueda se realizó de acuerdo con los principios PRISMA, resultando una selección preliminar de 21 estudios que fueron sometidos a una validacion de calidad utilizando el instrumento Quality Assesment Tool, que incluyo finalmente 14 estadios. Tres preguntas gulas se plantearon para realizar el analisisis zque características tiene la interacción social entre ninos con NEE $y$ sus pares? ?qué beneficios y limitaciones ofrecen las escuelas regulares para ninos con Neser y

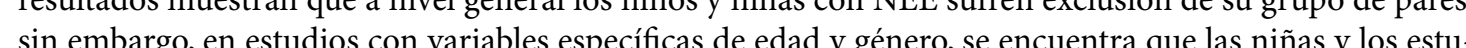
diantes de edades tempranas muestran actitudes más positivas hacie sub compañeros con NFE Adestr-

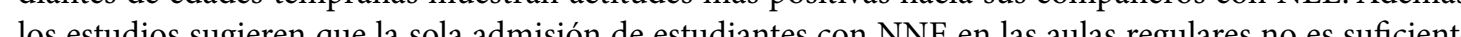
para lo pacentes yestudintes plo

Palabras Clave: INTERACCIÓN SOCIAL, NECESIDADES EDUCATIVAS ESPECIALES, INCLUSIÓN EDUCATIVA

\section{ABSTRACT}

Social Interaction Among Boys and Girls with Special Educational Needs and Their Peers A Narrative Review

This study seeks to analyze the social relationships between boys and girls with Special Educational Needs (SEN) and their peers, using a narrative review as a method of analysis of articles related to the topic preliminary selection of 21 studies, validated through the Quality Assessment Tool which resulted in the final consideration of 14 studies. Three questions guide the analysis, what are the characteristics of socil interaction between boys and girls with SEN and their peers? what are the benefits and limitations the regular education settings offer for children with SEN? and how do peer attitudes relate to learning? The results show that in general, boys and girls with SEN experience exclusion from their peer group; however, specific variables of are and gender, show that girls and early-age students are more willing to de hower, more positive attitudes towards their peers with SEN. In addition studies suggest that the mere admission of students with SEN in regular schools is not enough to achieve full inclusion, it is necessary a permanent support, interventions and training for teachers and students in regular schools.

Keywords: SOCIAL INTERACTION, SPECIAL EDUCATIONALNEEDS, LEARNING INCLUSION 
educativa requiere más que políticas in clusivas para los estudiantes con NEE. Po ejemplo, Allport (1954), explica que, si lo miembros de un grupo tienen un rol o estatus inferior, es probable que se refuercen los estereotipos existentes, el contacto con individuos o grupos de un rol o estatu superior puede producir sentimientos $\mathrm{d}$ inferioridad. Después de varias investigaciones con estos grupos, Allport construye la teoría de contacto, en la cual establece que el contacto con miembros diferentes a uno mismo conducirá a un cambio de actitud si se presenta en las condicione adecuadas.

En este sentido, la teoría de contacto en el aula de clase por un lado puede fomentar los contactos sociales y las relaciones con pares que se desarrollan normalmente, lo que permite que puedan convivir con la diversidad, aceptando las diferencias de cada compañero/a, las relaciones sociales positivas en el aula no favorecen únicamente a los estudiantes con NEE, pues los alumnos regulares también se benefician, ya que pueden desarrollar conductas pro-sociales, empatía, tolerancia y respeto por la diferencia, al punto que disminuyen las conductas agresivas (González-Rojas y Triana-Fierro, 2018). Pero de otro lado, este contacto puede generar que los estudiantes con NEE se sientan solitarios, con menos amigos que sus pares con mayor probabilidad de ser víctimas de acoso escolar; estas experiencias sociale negativas pueden afectar el autoconcepto y su visión del mundo los rodea, esta exclusión educativa constituye generalmente la antesala de la exclusión social (Koller, Pouesard y Rummens, 2017).

Además, algunas veces los niños y niñas con NEE presentan conductas agresivas o distintas a la mayoría de los miembros del salón de clase, lo cual puede ocasionar frustración. Se evidencia entonce una necesidad para responder ante situaciones negativas, en el manejo de las emociones y en la resolución de conflictos. E ocasiones los niños con discapacidad inte-

lectual presentan comportamientos agre- sivos que no facilitan las relaciones con los pares en la escuela (Cabrera-García, Lizarazo-Sandoval y Medina-Casallas 2016).

Así también cuando los docente cuentan entre sus alumnos estudiantes con NEE, sus actitudes hacia ellos pueden verse influenciadas por diversos fac tores, es posible que no se encuentren lo suficientemente capacitados para dar respuesta a dichas necesidades, pueden esta a cargo de grupos numerosos de estudiantes, contar tes, contar con escasos materiales de trabajo, las creencias respecto al rendimiento académico están basadas en las características de los niños, en los sentimientos que provocan en ellos y en su capacidad para aprender (Gavia, 2013; González-Rojas y Triana-Fierro, 2018) por esto, el docente está llamado a asumir un papel protagónico, pues en sus prácticas pedagógicas e el responsable de que todos los alumno alcancen el desarrollo de competencias cognitivas y sociales.

En cuando a los padres y madres de familia de estudiantes con NEE, se ha encontrado que no conocen con certeza si el ambiente de educación en las escuela regulares es constructivo o acoge de manera adecuada a sus hijos e hijas; de hecho, existen opiniones divididas entre si es positivo o negativo ya que por una parte es un derecho, pero al mismo tiempo no existen garantías de que reciban la educación acorde a sus necesidades particulares. En la mayoría de los estudios se muestra que los padres tienen actitudes positivas, aunque manifiestan preocupación por la falta de servicios individualizados $y$ refalta de servicios individualizados y recursos que puedan atender las necesida-
des puntuales de los estudiantes con NEE, también es necesario tomar en cuenta la implicación de factores afectivos y sociales (Jaén, 2017; Seade, Peñaherrera-Vélez, Huiracocha y Vélez-Calvo, 2019).

Las consecuencias de las actitudes negativas por parte de los compañeros podría resultar perjudicial para los niños y niñas con NEE, algunos de los principales efectos de la exclusión social en el entorno escolar incluyen agresividad, deserción vas Especiales (NEE) podrían presenta

los aprendizajes aun cuando se maneje la
Personas con Discapacidad 2006; Ley Or-

La literatura sugiere que la inclusión 
acoso escolar, problemas psicológicos, desmotivación para asistir a la escuela, problemas de autonomía, inseguridad, agresividad y miedo a afrontar retos que se le presenten en su diario vivir. Estas tres últimas condiciones en ocasiones persisten hasta la edad adulta (Castillo y Caicedo, 2012; Garcés, 2004; Parada, 2005).

Frente a esta situación este trabajo de investigación se centra en artículos científicos relacionados al tema de la interacción social entre pares en el ámbito educativo regular para establecer ¿qué características tiene la interacción social entre niños y niñas con NEE y sus pares? ¿qué beneficios y limitaciones ofrecen los ambientes de educación regular para niños con NEE? $\mathrm{y}$ ¿cómo inciden las actitudes de los pares en el aprendizaje?

\section{Materiales y Métodos}

El método de análisis usado es la revisión narrativa que de acuerdo a Enferm (2007), es un método de estudio que describe y analiza el estado de la ciencia de un tema específico desde un punto de vista teórico y contextual; además, consiste en un análisis crítico de la literatura publicada en libros y artículos de revistas electrónicas o en papel. Estas revisiones, son muy importantes porque proporcionan datos útiles a los lectores sobre temas específicos.

En un inicio se desarrolló una estrategia de búsqueda utilizando las palabras: inclusión educativa AND exclusión; actitudes de pares AND necesidades educativas; Peer attitudes AND social interaction; Attitudes students towards peers AND special educational needs; relaciones sociales AND necesidades educativas especiales. Las búsquedas se realizaron en siete bases de datos: Taylor and Francis, Springer, Latindex, Scielo, Redalyc, Science Direct y Psicinfo. Los criterios de exclusión primarios fueron: artículos de revisiones narrativas y meta análisis; estudios realizados en escuelas especiales, centros de salud, atención psicológica: en participantes mayores de doce años, adultos, docen- tes y familia (Tabla 1). Los resultados se analizaron de acuerdo con los principios PRISMA (Figura 1).
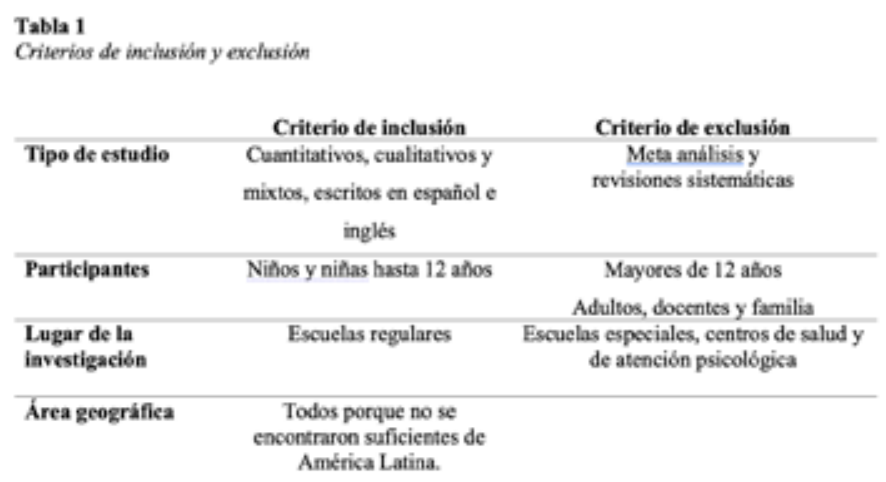

Las búsquedas iniciales arrojaron un resultado de 157 artículos. Después de la selección se eligieron veintiún artículos. selección se eligieron veintiun artículos.
Para ello se utilizó el diagrama de flujo PRISMA (figura 1), este instrumento es un conjunto mínimo de elementos basados en evidencias para informar en revisiones sistemáticas y metanálisis; se centra en el informe de revisiones que evalúan ensayos aleatorios, pero también se puede utilizar como base para informar revisiones bibliográficas de otros tipos de investigación (The PRISMA Group, 2009).

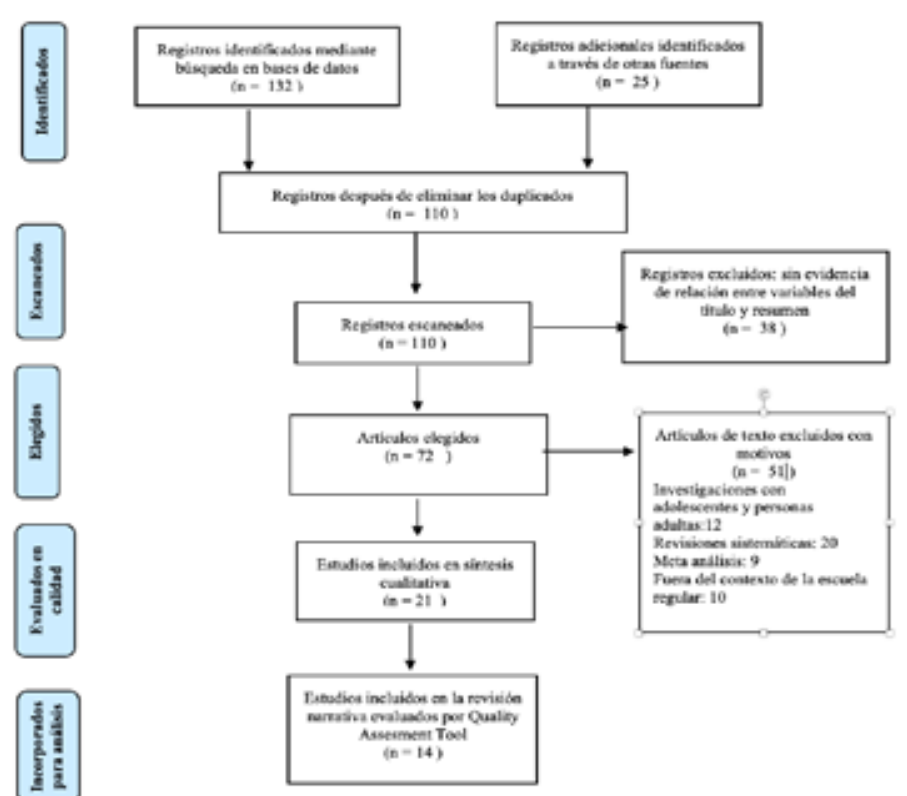

Figura 1. Diagrama de selección de artículos. Fuente: PRISMA
En la tabla 2 y 3 se describen la validación de los 21 artículos seleccionados. E instrumento que se utilizó fue Quality Assesment Tool como criterios de evaluación de calidad estándar para analizar artículos de investigación primarios de una variedad de campos; este instrumento ayuda a evalua la calidad de los informes de investigación primarios, mediante una lista de verificación que se diseñó específicamente para su uso con estudios cualitativos y cuantitativo (Kmet, Lee y Cook, 2004).

En la lista de verificación del instrumen-

Tabla 2

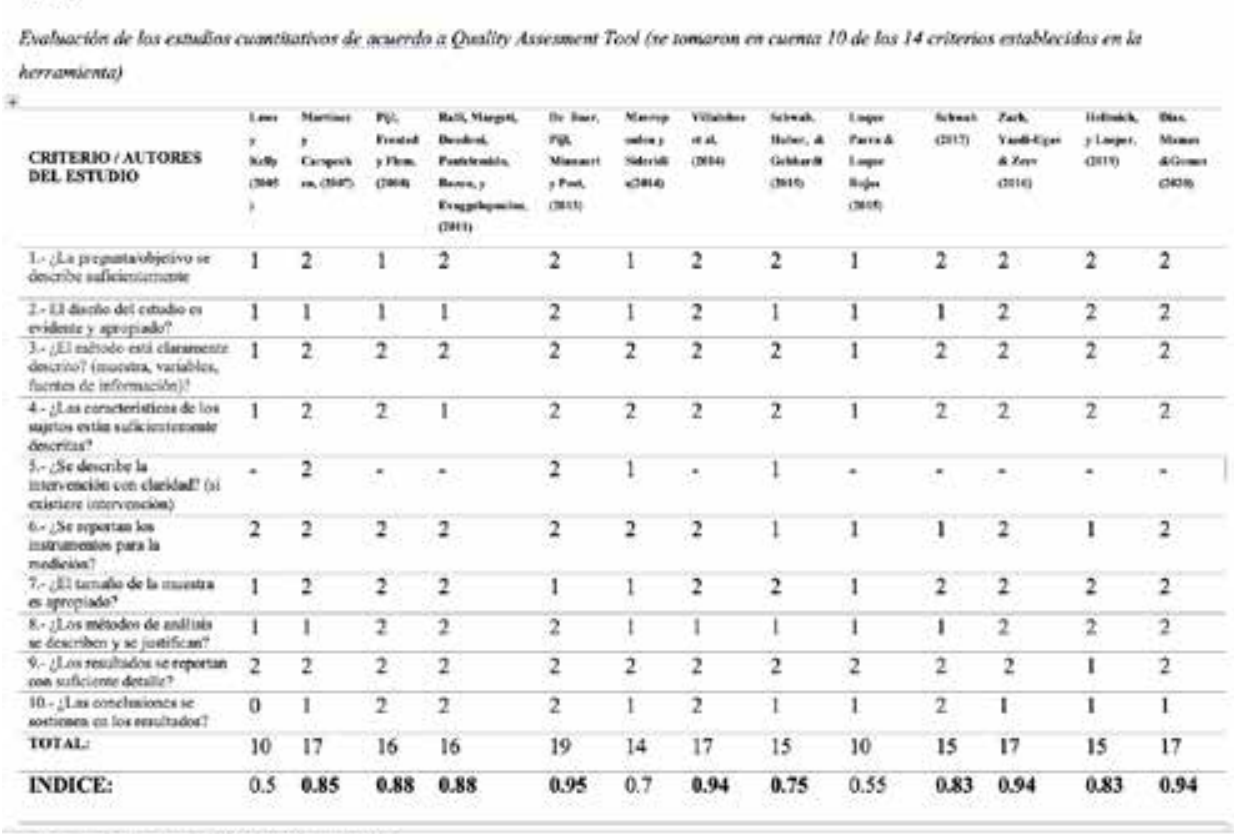

Tabla 3.

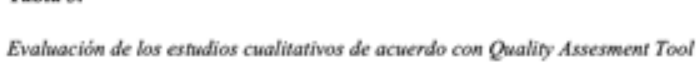

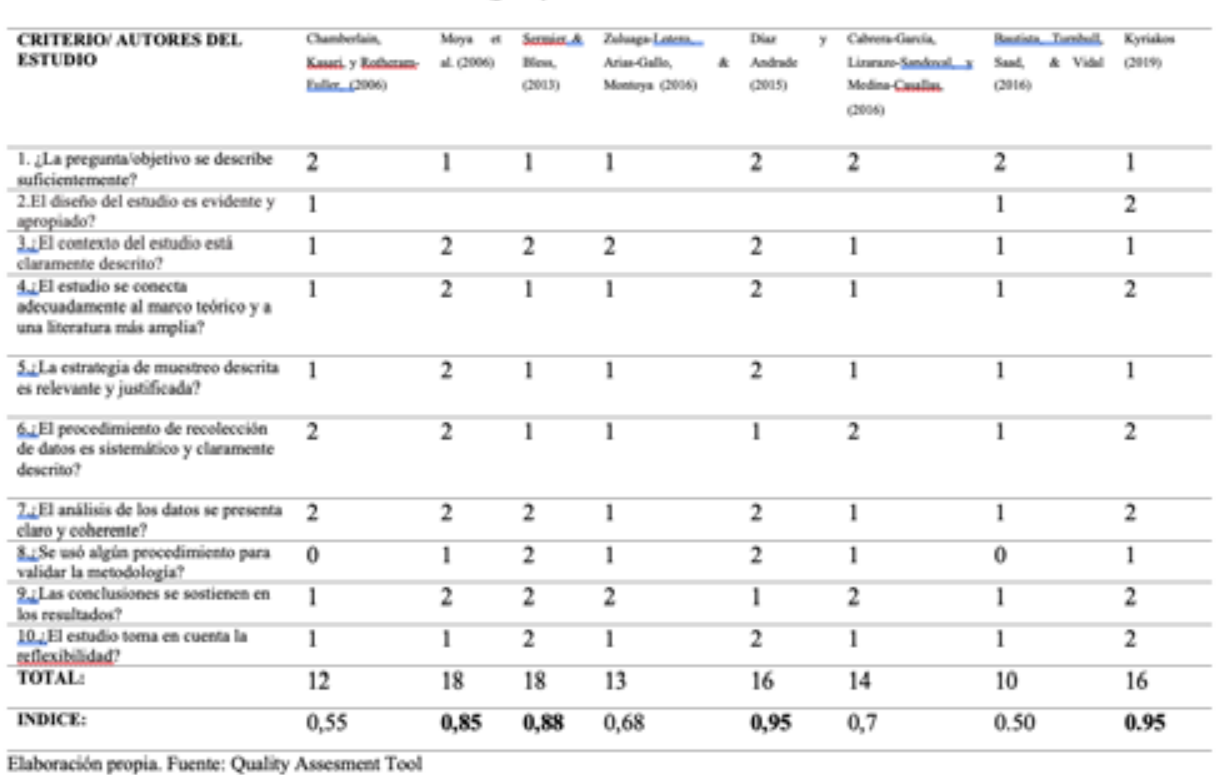

to Quality Assesment Tool, se evaluó criterios importantes para la validación de los artículos, entre ellos: objetivo, diseño del estudio, muestra, recolección y análisis de datos, metodología, resultados y conclusiones. Como resultado de la validación y de acuerdo con los criterios establecidos, se excluyeron todos los estudios que arrojaron una valoración inferior a 0.70 seleccionándose entonces los 14 estudios que cumplieron con los puntajes entre 0,70 y 1,00 para el análisis narrativo en base a las tres preguntas guías de la investigación. 


\section{Análisis y discusión}

Características de la interacción social entre niños con NEE y sus pares

Los estudios analizados refieren a aspectos no muy alentadores en cuando a la interacción social y a la convivencia en e aula entre estudiantes con y sin NEE, varios trabajos refieren a que los niños y niñas con NEE no tienen relaciones positiva con los compañeros. Generalmente, son estudiantes invisibles en el aula con un posición que oscila entre la marginación el rechazo porque son percibidos en form negativa por sus pares que los identifican como quienes manifiestan conductas distractoras en la clase (Moya et al., 2006). Villalobos et al. (2014) evaluaron exclusivamente la convivencia en la escuela, y encontraron que aquellos estudiantes, que fueron diagnosticados con alguna necesidad educativa especial o que reciben apoyo pedagógico, son víctimas de más agresiones en los entornos educativos regulares. Además, este grupo de estudiantes es más propenso a ser víctima de agresión social en un $58,2 \%$ comparado con el 36,50\% en sus pares sin NEE. El estudio de Pij Frostad y Flem (2008) encuentra tambié que los niños con NEE están excluidos de su grupo de pares, son menos populares, tienen menos amistades y participan con menos frecuencia como miembros de un subgrupo. Adicionalmente, tienen mayo posibilidad que sus pares sin NEE de ubicarse en un grupo de riesgo. Los autores también dan cuenta de un sobre-optimismo de los docentes con respecto a las relaciones en el aula entre pares en relación lo que realmente ocurre entre estudiantes con y sin NEE. Para el caso de la inclusión de niños con síndrome de Down (SD) Schwab, Huber y Gebhardt (2015) describen que los niños con este diagnóstico son en general menos aceptados socialmente que los niños sin discapacidad y que las intervenciones basadas en la retroalimentación de los docentes hacia el grupo de es- tudiantes, en cuanto a la aceptación social os niños con discapacidad, es importante.

Pero conforme los estudios van alcanzando un nivel menos general y se enfocan en género o edad, aparecen diferencias en las actitudes grupales hacia erencias en las actitudes grupales hacia estudiantes con NEE. En cuanto a género, por ejemplo, el estudio de Ralli et al. (2011) encuentra que más niñas que niños expresaron actitudes positivas hacia ayudar a un compañero con dificultades de prendizaje y en la misma línea argumentativa, para Martínez y Carspecken (2007) en ambientes multiculturales, los niños no interactúan de forma natural con los niños que tienen discapacidades a menos que se les anime a hacerlo, pero las niñas (particularmente hispanas) muestran actitudes más favorables en relación a niños con discapacidades antes y después de implementar tratamientos para promover la inclusión escolar.

En cuanto a edad, De Boer et al. (2013) dan cuenta de los resultados de un cuasiexperimento de un programa de intervención para influenciar las actitudes de los estudiantes de la escuela primaria hacia compañeros con discapacidades físicas e intelectuales; las mediciones realizada antes, durante y después de la intervención muestran resultados inmediatamente positivos en estudiantes de nivel preescoar pero no así en estudiantes de educación básica. Esta tendencia es corroborada po otro estudio que encuentra que a medida que aumenta la edad, los estudiantes presentan reacciones y actitudes emocionales más negativas hacia los niños con NEE.

De acuerdo con estos estudios se puede decir que las actitudes afirmativa emergen cuando se hacen particularizaciones en los grupos, así por ejemplo en cuanto a género y edad, además a nivel inaren las diferencias actit nales mucho más que a nivel grupal. Para Schwab (2017), los estudiantes con NEE son nominados con menos frecuencia por sus compañeros para actividades conjuntas y para trabajar colaborativamente en proyectos escolares, pero cuando son elegidos, la elección viene de estudiantes que tienen actitudes más positivas frente a personas con discapacidad. Estas actitudes positivas están muy relacionadas con el ambiente familiar de los niños y niñas. En la misma línea, el estudio de Helmich y Loeper (2019) encuentra que las actitudes constructivas por parte de estudiante hacia sus pares con NEE dependen de sus experiencias de contacto, de lo que creen en cuanto a la autoeficacia del otro y de las percepciones del comportamiento de sus padres hacia personas con NEE. Al parecer la aceptación y las actitudes positiva están relacionadas con otros factores a nivel extra-escolar que inciden en la aceptación de personas con discapacidad o NEE.

La amistad entre niños en etapa de desarrollo y sus compañeros con discapacidades físicas se forma sobre la base de lo que los niños entienden y sienten acerca de las discapacidades que reflejan los estereotipos sociales del ambiente propio en e que los niños crecen. Es así como se hace necesario que los niños vivan la diversidad y la inclusión social como experiencias de primera mano, de la vida cotidian (Kyriakos 2019). El trabajo de Ralli et al. (2011) aborda las formas como los niños entienden la diversidad, identificando que la mayoría basan las diferencias a nive individual, en las diferencias biológicas y mucho menos en las diferencias por discapacidad. De otro lado, hay mucha desinformación y desconocimiento entre lo niños de las escuelas regulares respecto las causas de las dificultades para aprender que afectan a otras personas. Si bien se puede evidenciar que los niños se inclinan a ayudar, pueden tener alguno estereotipos negativos sobre convivir de manera cercana con personas con NEE en su escuela. Resumiendo, en cuanto a la interacción social, los estudios muestran que las actitudes negativas en cuanto a la interacción entre niños y niñas con y sin NEE se tornan evidentes a nivel grupal pero conforme se particulariza el enfoque se pueden encontrar hallazgos más alentadores. Son mediadores el entorno familia y el ambiente social más tolerante con la diversidad.

Beneficios y limitaciones que ofrecen los ambientes de edu cación regular para niños con NEE

Si bien es cierto que cuando se propicia la proximidad y contacto con los niños y niñas con NEE, los resultados muestran que las actitudes son más favorables en las dimensiones afectivas, cognitivas y conductuales (Dias, Mamas y Gome 2020), este contacto tiene que ser espontáneo porque cuando se elige libremente la interacción con un compañero con NEE se propician actitudes más positivas hacia la discapacidad (Schwab, 2017). En el caso de las escuelas inclusivas, muchas veces esta interacción libre no se logra, de hecho, Schwab et al. (2015) no encuentran diferencias en cuanto a la inclusión de estudiantes con SD en escuelas inclusivas y regulares; inclusive, la taza de aceptación social fue mayor en escuelas regulares que en las escuelas inclusivas.

En cuanto a la relación entre el contacto y las actitudes en escenarios escolares convencionales donde asisten estudiante con NEE, Schwab (2017) encuentra que la asistencia a la misma aula de niños y niñas con y sin NEE no tiene ningún efecto positivo notable pero podría tener efectos negativos en las actitudes de los estudiantes; es más, no se encontraron diferencias en las actitudes de estudiantes frente a sus pares con NEE entre aulas inclusivas y regulares. En este sentido, la presencia física de los niños y niñas con NEE en las escuelas regulares es solo un paso que necesita otros aspectos de apoyo para que puedan ser realmente incluidos en el grupo (Pijl el al., 2008).

El estudio de Díaz y Andrade (2015) explora las perspectivas los profesores, que en general ven que las actitudes que presentan los niños con Trastorno del Espectro Autista (TEA) tienen repercusiones para si mismos con mediana incidencia 
en la tensión en el aula. La gran mayoría coincide en la necesidad de capacitación y asesoría a los docentes. De Boer et al. (2013) dan cuenta de que los programas de intervención para influenciar las actitudes de los estudiantes pueden dar resultados positivos, pero no del mismo modo en diferentes grupos etarios. Dias et al. (2020) y Martínez y Carspecken (2007) afirman que las actitudes hacia las personas con discapacidades se desarrollan en la infancia y están influenciadas por las creencias de los padres y los profesores.

Para Villalobos et al. (2014) existe evidentes circunstancias de desventaja $\mathrm{d}$ los estudiantes con NEE al ser víctima de agresión con mayor frecuencia que sus pares sin diagnóstico de NEE. Frente a estas circunstancias las acciones que tom el sistema educativo es castigar los comportamientos agresivos, pero se hace mu poco para prevenirlo; las actividades administrativas y pedagógicas reciben mayo atención que la dimensión social y la plena inclusión en la comunidad educativa. En este sentido, son necesarios insumos adicionales al contacto entre estudiantes con y sin NEE. Las intervenciones muestran ser eficaces para influir positivamente en las actitudes de los niños hacia sus compañeros con NEE (Martínez y Carspecken, 2007; De Boer et al., 2013). Por otro lado, los docentes expresan que la mejora en las habilidades sociales mejoraría logros académicos y relaciones con docentes y compañeros (Díaz y Andrade 2015).

Si bien se han logrado avances en temas de inclusión en los ambientes escolares, aún hay mucho camino que recorrer. Al parecer, son necesarios programas de apoyo dentro de las escuelas inclusivas.

Incidencia de actitudes entre

pares en el aprendizaje

Entre los artículos analizados no se encontraron estudios que analicen específicamente la incidencia de las actitudes entre pares y el aprendizaje de estudiantes con NEE. Zach et al. (2016:37) compara- ron estudiantes con y sin diagnóstico de trastornos de aprendizaje, y encontraron que los logros académicos y los problemas de conducta predicen el 70\% de las habili政 trastornos de aprendizaje. Este hallazgo es alentador al mostrar que los niños con deficiencias leves se integran en las clases regulares cuando se llevan adelante intervenciones: "los programas desarrollados para mejorar el funcionamiento socioemocional de los estudiantes y los servicios de prevención dan como resultado una mejora tanto en los aspectos sociales como en los logros académicos".

Frente a este tema se sostiene un debate porque otros estudios encuentran que estudiantes con NEE tienen menos logros académicos (Zach et al 2016), son nominados con menos frecuencia por sus compañeros para actividades conjuntas, como trabajar juntos en un proyecto escola (Schwab, 2017) participan con menos frecuencia como miembros de un subgrupo (Pijl et al., 2008). Sin embargo, lo que se ha encontrado es que la inclusión de niños con discapacidad intelectual en las aulas de educación regular no muestra diferencia significativa en el progreso de estudiantes con alto o bajo rendimiento, ni que tampoco tenga un impacto negativo en rendimiento académico de los estudiantes sin discapacidad (Sermier y Bless, 2013).

\section{Conclusión}

La revisión narrativa que ha cubier to este artículo no es en modo alguno exhaustiva; sin embargo, ofrece pistas sobre as condiciones en las que se da la inclusión de estudiantes con NEE en los espacios de educación regular. Aun cuando varios estudios encuentran aspectos no muy alentadores en cuanto a la interacción socia cialy estudiantes on y sin NEE, en evidente desventaja para los primeros, se ha encontrado que al particularizar el análisis por género o edad se pueden identificar actitudes más positivas hacia estudiantes con NEE, especialmen- te entre las niñas y en grupos de edade más tempranas. Otro aspecto importante que recalcan los autores es el rol de la familia y el entorno general de la sociedad que acoja a las personas con discapacidad bajo condiciones de tolerancia y de san convivencia para que los niños desde tempranas edades crezcan en ambientes realmente inclusivos y repliquen estos comportamientos en el aula. Como señalan Peñaflor y Jara (2009), la educación puede ser un recurso importante para combati la discriminación, la exclusión y cimenta las bases de la inclusión, pero es necesari también un entorno macro social más favo rable que valore la diversidad como fuente y recurso del desarrollo que genere espacios abiertos para el diálogo, la convivencia y la construcción de la equidad.

Por otra parte, las políticas de vario países defienden y garantizan la inclusió educativa; sin embargo, de acuerdo con lo encontrado en esta revisión, el acceso de los estudiantes con NEE en aulas regulares no garantiza una plena inclusión educativa, son necesarios otros apoyos. Si bien el contacto con los estudiantes con NEE puede resultar beneficioso para sus compañe ros ya que les permiten convivir y conoce las diferencias, al mismo tiempo se mues- tra que para una convivencia armónica en el aula son necesarias intervenciones que permitan mejorar el conocimiento acerca de las características de cada NEE. Este recurso no necesariamente es aplicado por las instituciones educativas quienes principalmente brindan apoyos individualizados para estos estudiantes centrados en el aspecto cognitivo y administrativo institucional

Como limitación a este estudio cabe reconocer que no se pudo hacer una diferenciación entre estudios por región o por país para poder comparar si las condiciones de inclusión presentan variaciones notables. En este sentido, es necesario e importante plantear investigaciones que evalúen los alcances y limitaciones de las prácticas localizadas a nivel micro y de las políticas de inclusión educativa a nivel macro en Ecuador, ya que la mayoría de los artículos seleccionados han sido realizados en países europeos y otros escasos estudios realizados en Latinoamérica especialmente provenientes el cono sur. Quedan planteadas guías para otros trabajos que profundicen en la importanci de la familia y del entorno inmediato en la formación de actitudes más positivas frente a personas con NEE. 


\section{Referencias bibliográficas}

Allport,G.W.(1954). The nature of prejudice. Reading, MA Addison-Wes-ley.

Bautista, M., Turnbull, B., Saad, E., \& Vidal, A.(2016). La interacción de alumnos con discapacidad y sus pares en la escuela regular. Psicología Iberoamerican $24(1), 8-18$

Cabrera-García, V., Lizarazo-Sandoval, F. y Medina-Casallas, D. (2016). Necesidades de relaciones sociales de niños y niñas con discapacidad intelectual en la familia y en la escuela. Revista educación y desarrollo social. 10(2), 86-101. DOI: http://dx.do org/10.18359/reds.1554

Castillo, E. y Caicedo, J. (2012). Ponencia presentada en la Conmemoración Día Mundial Contra el Racismo "Por una educación libre de discriminación: del conocimiento formal a la garantía real". Bogotá.

De Boer, A., Pijl, SJ, Minnaert, A. y Post, W. (2013). Evaluating the Effectiveness of an Intervention Programto Influence Attitudes of Students Towards Peers with
Disabilities J Autism Dev Disord 44, 572-583 (2014). Disabilities J Autism Dev Disord 44,572-583
https://doi.org/10.1007/s10803-013-1908-6

Dias, P., Mamas, C. \& Gomes R. (2020): Attitudes of students towards peers with special educational needs in mainstream Portuguese schools, Euro-
pean Journal of Special Needs Education, DOI: pean Journal of Special Needs

Díaz, E. y Andrade, I. (2015). El Trastorno del Espectro Autista (TEA) en la educación regular: estudio realizado en instituciones educativas de Quito, Ecuador. Revis163-181 ISSN: 0187-7690. Dispo 163-181 ISSN: 0187-7690. Disponible en: https:/

Enferm, P. (2007). Revisión sistemática de la literatura y re visión narrativa. Acta Paul Enferm, 20,2

Faas, A. (2017).Psicología del desarrollo de la niñez. Retrieved from https://ebookcentral.proquest.com

Garcés, D. (2004). Aproximación a la situación educativa afrocolombiana. Estudios afrocolombianos aportes para un estado del arte (pp. 147-175). Popayán: Universidad del Cauca

Gavia, P. (2013). Creencias de los profesores sobre los problemas de conducta en alumnos con discapacidad blemas de conducta en alumnos con
intelectual. Ra Ximhai, $9(4), 245-258$.

González-Rojas, Y. \& Triana-Fierro, D. A. (2018). Actitudes de los docentes frente a la inclusión de estudiantes con necesidades educativas especiales. Educación con necesidades educativas
Educadores, 21(2), 200-218.

Hellmich, F. y Loeper, M. (2019). Children's attitudes towards peers with learning disabilities - the role of perceived parental behaviour, contact experiences and self-efficacy beliefs. Revista Británica de Educación Especial. doi: 10.1111 / 1467-8578.12259

Jaén, A. (2017). La inclusión educativa desde la voz de los padres. Revista de Educación Inclusiva, $7(1)$

Laws, G., \& Kelly, E. (2005). The attitudes and friendship intentions of children in United Kingdom mainstrea schools towards peers with physical or intellectual disabilities. International Journal of Disability, Development and Education, 52(2), 79-99.

Ley Orgánica de Educación Intercultural LOEI (2001). Re cuperado de: https://educacion.gob.ec/documento legales-y-normativos/

López, S. y Valenzuela, B. (2015). Niños y adolescentes con necesidades educativas especiales. Revista Médica Clínica Las Condes, 26 (1), 42-51. https://do org/10.1016/j.rmclc.2015.02.004

Luque Parra, D, \& Luque Rojas, M. (2015). Relaciones de amistad y solidaridad en el aula: Un acercamiento psicoeducativo a la discapacidad en un marco inclusivo. Revista mexicana de investigación educativa, 20(65), 369-392.

Kyriakos D. (2019). Intentions of children without disabilities to form friendship with peers with physical disability: a small scale study, Early Child Development and Care, Do1. 10.1080/030440.2019.1697246

ooller, D., Pouesard, L., \& Rummens, A. (2017). Defining Social Inclusion for Children with Disabilities: Critical Literature Review. Children \& Society, 32(1)

Macchiarola, V. y Perassi, Z. (2018). Políticas de inclusión educativa: la evaluación del impacto de algunas experiencias en Argentina. Miño y Davila. https//4
bronent/es/ereader/utnorte/116980?page 17

Martínez, R. y Carspecken, P. (2007). Efectividad de una intervención breve sobre la aceptación social de los nños latinos de sus compañeros con necesidades espropoulou, S. Y Sideridis G. (2014) Knowledge of Attism and y sidid of tially Ind sorders J Autism Dev Disord 44, 1867-1885 (2014). https://doi.org/10.1007/s10803-014-2059-0

Moya, A., Romero, C., Macías, Á., Gómez C., y Moreno N. (2006). Necesidades educativas especiales en las aulas ¿compañeros y compañeras invisibles? En-clave pedagógica, 8 .

Organización de las Naciones Unidas ONU (2006). Convención de los Derechos de las Personas con Discapacidad.

Parada, D. (2005). Percepciones sobre el territorio y su relación en la enseñanza de las ciencias sociales desde una perspectiva de educación intercultural a partir ñas de orign afrocos Cordova del municipio de San Onofre, Sucre (tesis de maestría), Universidad de Antioquia, Medellín

Peñaflor G y Jara L (2009). Representaciones populares torno a la discriminación. Lima: Centro de Estudios y Publicaciones, Instituto Bartolomé de Las Casas.

Pijl, S., Frostad, P. y Flem, A. (2008). The social position of pupils with Special Needs in regular schools. Revista escandinava de investigación educativa, 52 (4)
387-405.

Ralli, A., Margeti, M., Doudoni, E., Pantelemidou, V., Rozou, T. y Evaggelopoulou, E. (2011). Typically developing children's understanding of and attitudes towards diversity and pecrs with learning difficulties in the Greek setting. European Journal of Specis Needs Educaion, 26 (2), 233-249. doi: $10.1080 /$

Schwab, S., Huber, C., \& Gebhardt, M. (2015). Social acceptance of students with Down syndrome and student without disabilty. Educational Psychology, 36(8)

Schwab, S. (2017). The impact of contact on students' attitudes towards peers with disabilities. Investigació en discapacidades del desarrollo, 62, 160-165. do: 10.1016/j.ridd.2017.01.015

Seade, C., Peñaherrera-Vélez, M. J., Huiracocha, K., y VélezCalvo, X. (2019). Actitudes hacia la educación inclusiva de los padres de niños con discapacidad. Educación en Contexto, Vol. VI, $\mathrm{N}^{\circ}$ Especial, Julio, 2020. 477-9296

Sermier R., \& Bless, G. (2013). The impact of includin children with intellectual disability in general education classrooms on the academic achievement their low-, average-, and high-achieving peers. Jour-
Defollectual \& Developmental Disability $38(1)$ 23-30

The PRISMA Group, (2009). Elementos de informe preferidos para revisiones sistemáticas y metaanálisis: declaración PRISMA. PLOS Med 6 (7): el000097. doil $10.1371 /$ jouna. Prodionog7

Classification of Education (ISCED).

Villalobos B., Carrasco C., López V., Bilbao M. Á., Morales M., Ortiz S., \& Ayala del Castillo, A. (2014). Inclusión y violencia. Prevalencia de victimización entre pares gráón Escolar. patro

ach, S., Yazdi-Ugav, O., \& Zeev, A. (2016). Academic achievements, behavioral problems, and lonelines and without learing disorders. School Pychology International, 37(4) 378-396. htps/ldoorg/10.1177/0143034316649231

Zuluaga-Lotero, A., Arias-Gallo, L., \& Montoya, M. (2016). Proyecto Mosqueteros: una propuesta de intervención en el aula para favorecer los procesos de inclusión escolar. Pensamiento Psicológico, 14(1),77-88. ISSN: 1657-8961. Disponible en: https://www.redalyc.org/articulo.oa?id=801/80144041006 